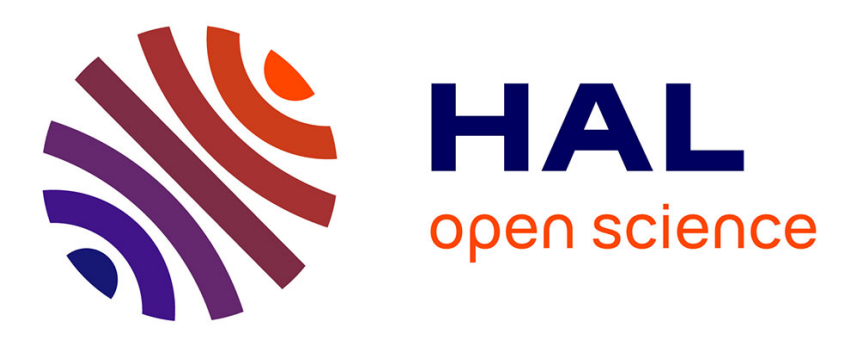

\title{
Analysis of Artifacts in Subpixel Remote Sensing Image Registration
}

Jordi Inglada, Vincent Muron, Damien Pichard, Thomas Feuvrier

\section{To cite this version:}

Jordi Inglada, Vincent Muron, Damien Pichard, Thomas Feuvrier. Analysis of Artifacts in Subpixel Remote Sensing Image Registration. IEEE Transactions on Geoscience and Remote Sensing, 2007, 45 (1), pp.254-264. hal-00578144

\section{HAL Id: hal-00578144 https://hal.science/hal-00578144}

Submitted on 18 Mar 2011

HAL is a multi-disciplinary open access archive for the deposit and dissemination of scientific research documents, whether they are published or not. The documents may come from teaching and research institutions in France or abroad, or from public or private research centers.
L'archive ouverte pluridisciplinaire $\mathbf{H A L}$, est destinée au dépôt et à la diffusion de documents scientifiques de niveau recherche, publiés ou non, émanant des établissements d'enseignement et de recherche français ou étrangers, des laboratoires publics ou privés. 


\title{
Analysis of Artifacts in Subpixel Remote Sensing Image Registration
}

\author{
Jordi Inglada, Vincent Muron, Damien Pichard, and Thomas Feuvrier
}

\begin{abstract}
Subpixel accuracy image registration is needed for applications such as digital elevation model extraction, change detection, pan-sharpening, and data fusion. In order to achieve this accuracy, the deformation between the two images to be registered is usually modeled by a displacement vector field which can be estimated by measuring rigid local shifts for each pixel in the image. In order to measure subpixel shifts, one uses image resampling. Sampling theory says that, if a continuous signal has been sampled according to the Nyquist criterion, a perfect continuous reconstruction can be obtained from the sampled version. Therefore, a shifted version of a sampled signal can be obtained by interpolation and resampling with a shifted origin. Since only a sampled version of the shifted signal is needed, the reconstruction needs only to be performed for the new positions of the samples, so the whole procedure comes to computing the value of the signal for the new sample positions. In the case of image registration, the similarity between the reference image and the shifted versions of the image to be registered is measured, assuming that the maximum of similarity determines the most likely shift. The image interpolation step is thus performed a high number of times during the similarity optimization procedure. In order to reduce the computation cost, approximate interpolations are performed. Approximate interpolators will introduce errors in the resampled image which may induce errors in the similarity measure and therefore produce errors in the estimated shifts. In this paper, it is shown that the interpolation has a smoothing effect which depends of the applied shift. This means that, in the case of noisy images, the interpolation has a denoising effect, and therefore, it increases the quality of the similarity estimation. Since this blurring is not the same for every shift, the similarity may be low for a null shift (no blurring) and higher for shifts close to half a pixel (strong blurring). This paper presents an analysis of the behavior of the different interpolators and their effects on the similarity measures. This analysis will be done for the two similarity measures: the correlation coefficient and the mutual information. Finally, a strategy to attenuate the interpolation artifacts is proposed.
\end{abstract}

Index Terms-Disparity map estimation, image registration, interpolation artifacts, similarity measures.

\section{INTRODUCTION}

$\mathbf{S}$ UBPIXEL accuracy image registration is needed for applications such as change detection [1], pan-sharpening [2], and data fusion [3]. In order to achieve this accuracy, the deformation between the two images to be registered is usually modeled by a displacement vector field which can be estimated by measuring rigid local shifts for each pixel in the image.

Manuscript received March 15, 2006; revised May 23, 2006. This work was supported by the Centre National d'Études Spatiales under Contract 4500013574/DCT094.

J. Inglada is with the Image Analysis Office, Centre National d'Etudes Spatiales, 31000 Toulouse, France.

V. Muron, D. Pichard, and T. Feuvrier are with the Space Division, Communications and Systémes, 31000 Toulouse, France.

Digital Object Identifier 10.1109/TGRS.2006.882262
In order to measure subpixel shifts, image resampling is used. Sampling theory says that, if a continuous signal has been sampled according to the Nyquist criterion, a perfect continuous reconstruction can be obtained from the sampled version. Therefore, a shifted version of a sampled signal can be obtained by interpolation and resampling with a shifted origin.

Since only a sampled version of the shifted signal is needed, the reconstruction needs only to be performed for the new positions of the samples, so the whole procedure comes to computing the value of the signal for the new sample positions.

In the case of image registration, the similarity between the reference image and the shifted versions of the image to be registered is measured, assuming that the maximum of similarity determines the most likely shift. The image interpolation step is thus performed a high number of times during the similarity optimization procedure. In order to reduce the computation cost, approximate interpolations are performed. Indeed, the ideal interpolator is a sinus cardinal function, and therefore, an infinite number of samples are needed for the computation of any new sample. Several approaches exist for reducing the computation time. For instance, a truncated sinc interpolator needs only a few samples. Other interpolators like the linear one can also be used.

Approximate interpolators will introduce errors in the resampled image which may induce errors in the similarity measure and therefore produce errors in the estimated shifts.

The problem of interpolation artifacts in image registration has been studied in the case of mutual-information-based registration in the context of medical images [5]-[7]. To our knowledge, there is no equivalent work in the field of remote sensing image registration. In this case, simple parametric geometrical transformations are not good candidates for deformation modeling. Also, similarity measures other than mutual information should be studied and characterized with respect to the interpolation artifacts.

Finally, the explanations given in the literature for the artifacts observed in registration functions do not seem to be satisfactory for all similarity measures and image modalities. Therefore, a theoretical modeling of the artifacts is proposed here. In this paper, we focus on the characterization of the artifacts for different similarity measures and interpolators, and we propose a theoretical explanation of the origin of the artifacts. Some guidelines and recommendations in order to attenuate these artifacts are also given.

The paper is organized as follows. In Section II, we pose the problem of subpixel image registration. In Section III, we show the effects of the artifacts on disparity maps with empirical tests. Section IV gives a theoretical explanation for the origin of the artifacts and checks its validity on test data. Section V 
TABLE I

APPROACHES TO IMAGE REgISTRATION

\begin{tabular}{|c|c|c|}
\hline Geometric model & Similarity measure & $\begin{array}{c}\text { Optimization of the } \\
\text { deformation }\end{array}$ \\
\hline Physical model & None & Global \\
\hline $\begin{array}{c}\text { Analytical model } \\
\text { with a priori HP }\end{array}$ & Local & Global \\
\hline $\begin{array}{c}\text { Analytical model } \\
\text { without a priori HP }\end{array}$ & Global & Global \\
\hline Grid & Local & Local \\
\hline
\end{tabular}

proposes strategies to attenuate the effects of the artifacts; and Section VI concludes the paper.

\section{Disparity-MAP ESTIMATION}

In this section, we recall the principle of disparity-map estimation used in order to achieve subpixel accuracy.

\section{A. Problem Position}

The problem of disparity-map estimation has been described in detail in [8]. We recall hereafter the main concepts involved with it.

The estimation of a disparity map between a reference image $I$ and secondary image $J$ can be posed as the following optimization problem:

$$
\operatorname{Arg} \max _{T}\left(S_{c}(I, T \circ J)\right)
$$

where $T$ is a geometric transformation and $S_{c}$ is a similarity measure: a scalar strictly positive function which has an absolute maximum when the two images $I$ and $J$ are identical in the sense of the criterion $c$.

In the case of image registration, the inverse of transformation $T$ is needed for image resampling.

Depending on the type of deformation to be corrected, the model used for $T$ will be different. For example, if the only deformation to be corrected is the one introduced by the mean attitude, a physical model for the acquisition geometry (independent of the image contents) will be enough. If the sensor is not well known, this deformation can be approximated by a simple analytical model. When the deformations to be modeled have high frequencies, analytical (parametric) models are not suitable for a fine registration. In this case, one has to use a fine sampling of the deformation, that means the use of deformation grids. These grids give, for a set of pixels of the reference image, their location in the secondary image.

We can thus conclude that the optimization of the similarity can be either global or local with a similarity measure, which can also be either local or global. All this is synthesized in Table I.

The ideal approach would consist of a registration which is locally optimized, both in similarity and deformation, in order to have the best registration quality. This is the case when deformation grids with dense sampling are used. Unfortunately, this case is the most computationally expensive, and one often uses either a low sampling rate of the grid or the evaluation of the

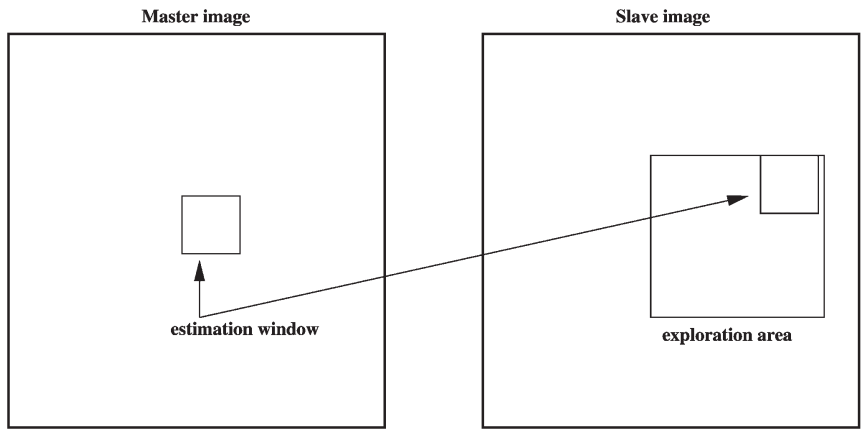

Fig. 1. Estimation of the similarity surface.

TABLE II

EXPRESSIONS OF FUNCTION $f$ IN THE $f$-DivERGENCE FAMILY

\begin{tabular}{|c|c|}
\hline Measure & $f(x)$ \\
\hline Kolmogorov distance & $\frac{1}{2}|x-1|$ \\
\hline Mutual information & $x \log x$ \\
\hline Kullback divergence & $(x-1) \log x$ \\
\hline$\chi^{2}$-divergence & $\frac{1}{2}(x-1)^{2}$ \\
\hline Hellinger distance & $\frac{1}{2}(\sqrt{x}-1)^{2}$ \\
\hline Toussaints distance & $x \frac{x-1}{x+1}$ \\
\hline Lin K-divergence & $x \log \frac{2 x}{1+x}$ \\
\hline
\end{tabular}

similarity in a small set of pixels for the estimation of an analytical model. Both of these choices lead to local registration errors which, depending on the topography, can amount several pixels.

Even if this registration accuracy can be enough in many applications (orthoregistration, import into a GIS, etc.), it may not be acceptable in the case of data fusion, multichannel segmentation, or change detection [1]. This is why we will focus on the problem of deformation estimation using dense grids.

As we have noted above, deformations due to topography can locally have high frequencies for medium- and high-resolution sensors (30 $\mathrm{m}$ and better), thus our need for fine modeling.

\section{B. Estimation Procedure}

The geometric deformation is modeled by local rigid displacements [8]. One wants to estimate the coordinates of each pixel of the reference image inside the secondary image. This can be represented by a displacement vector associated with every pixel of the reference image. Each of the two components (lines and columns) of this vector field will be called deformation grid.

We use a small window taken in the reference image, and we test the similarity for every possible shift within an exploration area inside the secondary image (Fig. 1). That means that, for each position, we compute the similarity measure. The result is a similarity surface whose maximum gives the most likely local shift between both images.

Quality criteria can be applied to the estimated maximum in order to give a confidence factor to the estimated shift: width of the peak, maximum value, etc. Subpixel shifts can be measured by applying fractional shifts to the sliding window. This is done by image interpolation. 


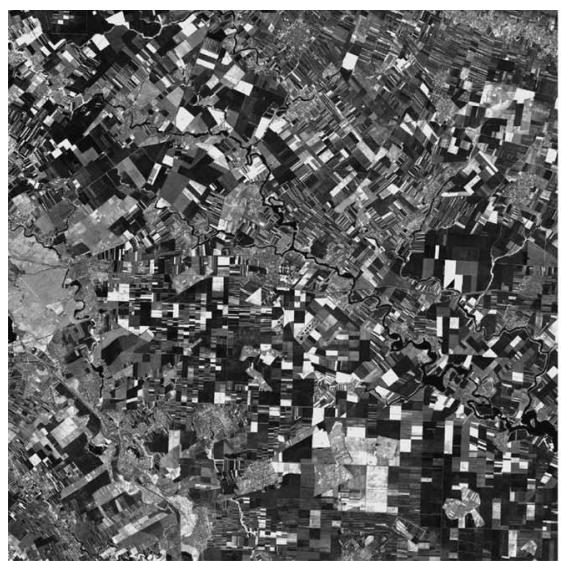

(a)

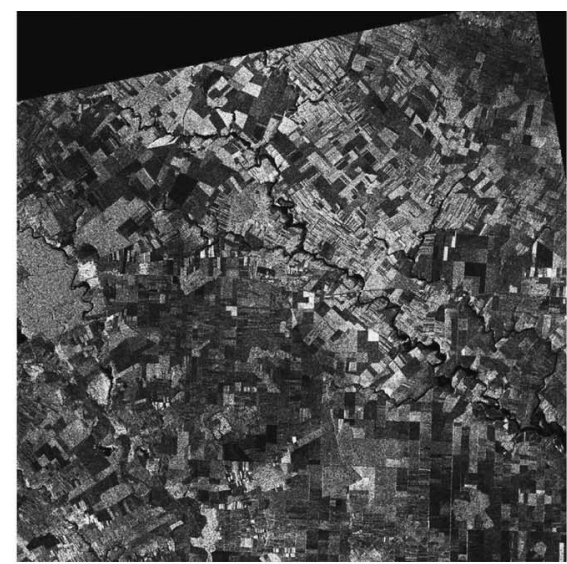

(b)

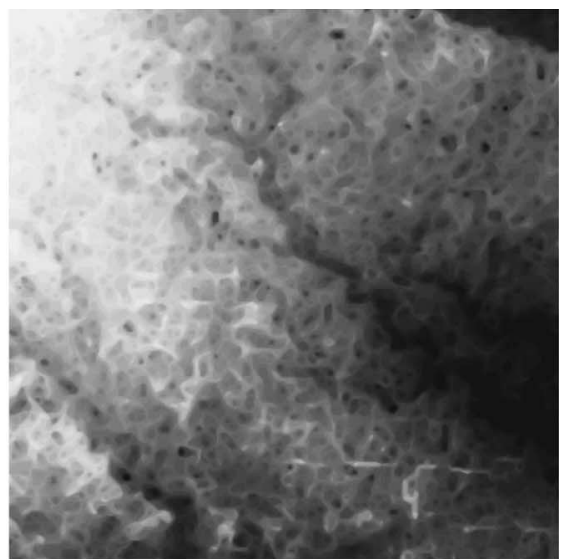

(c)

Fig. 2. Images and DEM for the test area. (a) Spot 4 B3. (b) ERS-2 SAR. (c) DEM.

The interesting parameters of the procedure are the following.

1) The size of the exploration area: it determines the computational load of the algorithm (we want to reduce it), but it has to be large enough in order to cope up with large deformations.

2) The size of the sliding window: the robustness of the similarity measure estimation increases with the window size, but the hypothesis of local rigid shifts may not be valid for large windows.

\section{Similarity Measures}

In this paper, we will only study two similarity measures: the correlation coefficient and the mutual information. A detailed discussion on similarity measures for image registration can be found in [8].

The correlation coefficient is the most used similarity measure for image registration. We remind here its computation for two image windows $I$ and $J$. The coordinates of the pixels inside the windows are represented by $(x, y)$

$$
\rho(I, J)=\frac{1}{N} \frac{\sum_{x, y}\left(I(x, y)-m_{I}\right)\left(J(x, y)-m_{J}\right)}{\sigma_{I} \sigma_{J}} .
$$

In this expression, $N$ is the number of pixels of the analysis window, $m_{I}$ and $m_{J}$ are the estimated mean values inside the analysis window of, respectively, images $I$ and $J$, and $\sigma_{I}$ and $\sigma_{J}$ are their standard deviations.

The mutual-information measure between two images can be understood as the amount of information we have from one image when we know the other. This is the degree of dependence between the two images. Several approaches exist for the computation of the mutual information. We prefer to introduce it as a member of the $f$-divergence family.

An $f$-divergence [9] measures the expectation of the diversity of the likelihood ratio between two distributions $P$ and $Q$

$$
D_{f}(P, Q)=E_{Q}\left[f\left(\frac{d p(x)}{d q(x)}\right)\right]=\int f\left(\frac{p(x)}{q(x)}\right) q(x) d x .
$$

$E_{Q}$ is the expectation with respect to $Q, d p(x) / d q(x)$ is the derivative with respect to a density, and $f$ is continuous and convex on $[0,+\infty)$. A divergence can be seen as a relative entropy. Depending on the choice of $f$, different measures can be obtained. Table II shows some interesting cases.

The mutual information between images $I$ and $J$ is the particular case where $f(x)=x \log x, p(x)=p_{I J}(i, j)$ (the joint probability density function of the two images) and $q(x)=$ $p_{I}(i) p_{J}(j)$ (the product of probability density functions of images $I$ and $J$ ). It can therefore be computed as follows:

$$
M I(I, J)=\sum_{i j} p_{I J}(i, j) \log \frac{p_{I J}(i, j)}{p_{I}(i) p_{J}(j)} .
$$

The sum is computed over every couple of pixel values $(i, j)$.

\section{Assessment of the Artifacts}

In this section, we introduce the problem of subpixel shift artifacts by analyzing the results obtained in a real case.

Our data set is consist of the following pair (a region of $2000 \times 2000$ pixels is used for our tests):

1) B3 channel of a SPOT 4 image (20-m pixel resolution) acquired on June 24, 2001, over the east of the Bucharest area [Fig. 2(a)];

2) European Remonte Sensing 2 (ERS-2) Satellite synthetic aperture radar three-look intensity image $(12.5-\mathrm{m}$ pixel size and approximately 20-m pixel resolution) acquired on May 10, 2001, over the same area [Fig. 2(b)].

Both images were orthorectified: For the SPOT 4 image, a digital elevation model (DEM) [Fig. 2(c)] with an altimetric accuracy better than $10 \mathrm{~m}$ and a planimetric accuracy around $10 \mathrm{~m}$ has been used together with the acquisition model (orbits, attitude) for the satellite; for the ERS-2 image, no DEM was used, but a constant altitude and homologous points manually taken on the SPOT 4 image were used in the orthorectification process. Globally, the images show a good superposition, but local errors exist, which can amount several pixels due to the simple geometric modeling of the deformation of the radar image.

If we analyze the DEM, we see that a gentle slope descending from northwest and southeast exists and that abrupt topography 


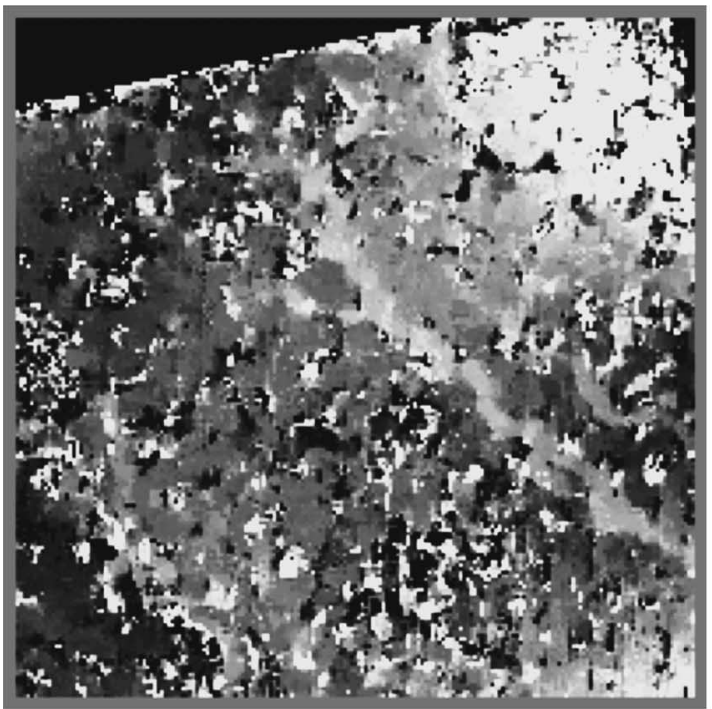

(a)

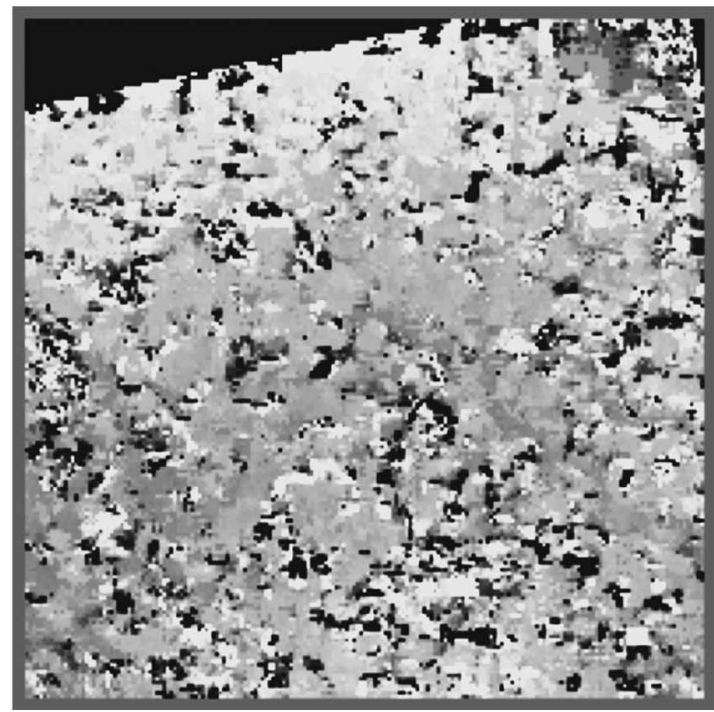

(b)

Fig. 3. Deformation grid. Mutual information: estimation window is $51 \times 51$ pixels, and sampling rate is five pixels. (a) Horizontal. (b) Vertical.

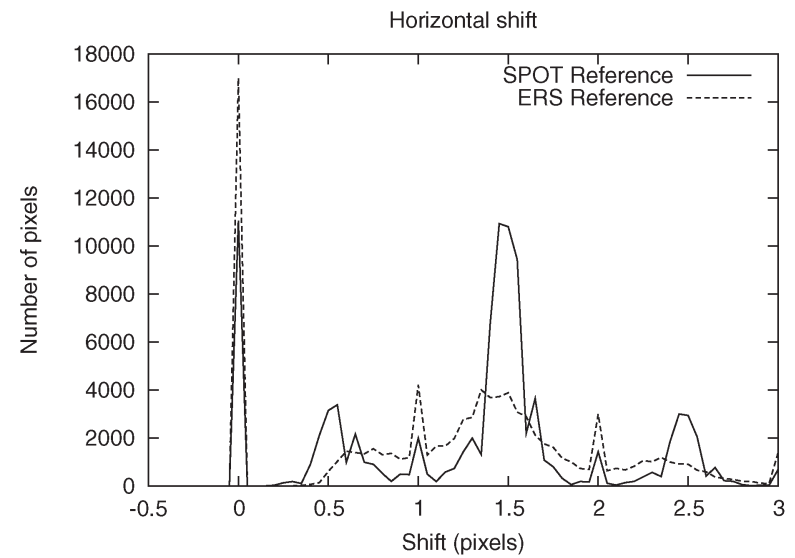

(a)

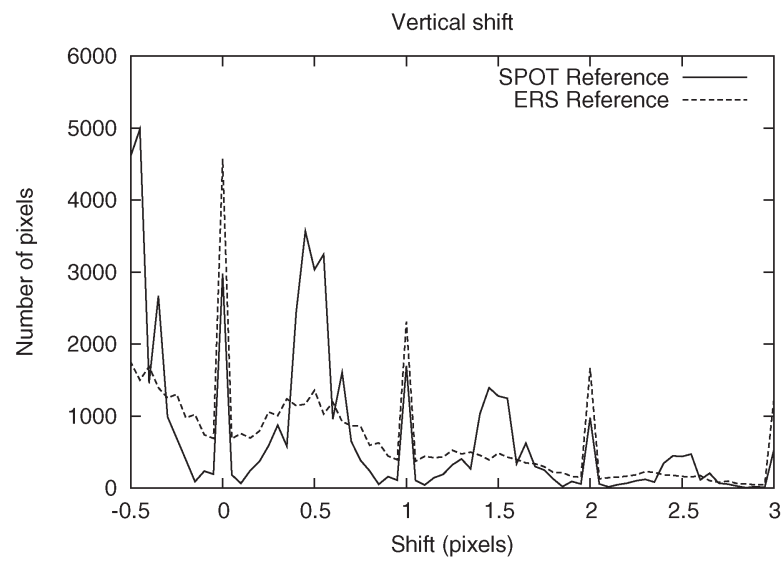

(b)

Fig. 4. Histograms of the estimated subpixel shifts [(a) horizontal and (b) vertical] with inversion of the reference and the secondary images.

features appear in the northeast and southwest. The shape of the river can also be identified in the DEM.

Fig. 3 presents the horizontal and vertical components of the displacement vector field obtained using the mutual information similarity measure and the procedure described in Section II-B. One observes a good correlation between the horizontal component and the topography shown in Fig. 2(c). As expected, the vertical (satellite along-track) direction does not show any particular structure. When this displacement vector field is used for the registration of the images, a good superposition is achieved. The detailed analysis of the procedure was carried out in [8].

If we analyze the distribution of the estimated shifts by computing their histograms, we observe the following behavior (Fig. 4). When the SPOT image is used as the reference, a high number of estimated shifts are multiples of 0.5 pixels; if the ERS image is used as the reference, this effect is attenuated, and the shifts present a more uniform distribution.

Since the similarity measure is the same for both cases and so is the optimization procedure, one can conclude that the subpixel shifts artifacts appear when the ERS image is

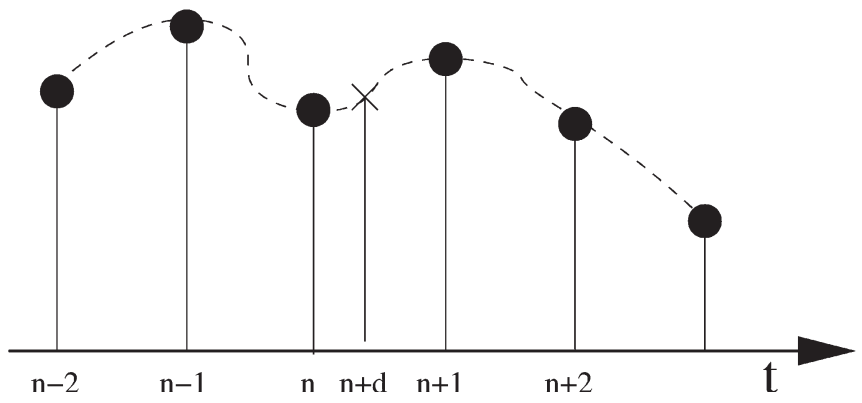

Fig. 5. Interpolation of a sampled signal.

interpolated during the similarity optimization. The following sections will study this effect in detail, and a theoretical model for the origin of the artifacts will be presented.

\section{ORIGIN OF the ARTIFACtS}

The problem of interpolation artifacts in the similarity surfaces has been studied for the case of mutual-information-based medical image registration [5]-[7]. Pluim et al. [5] show that 


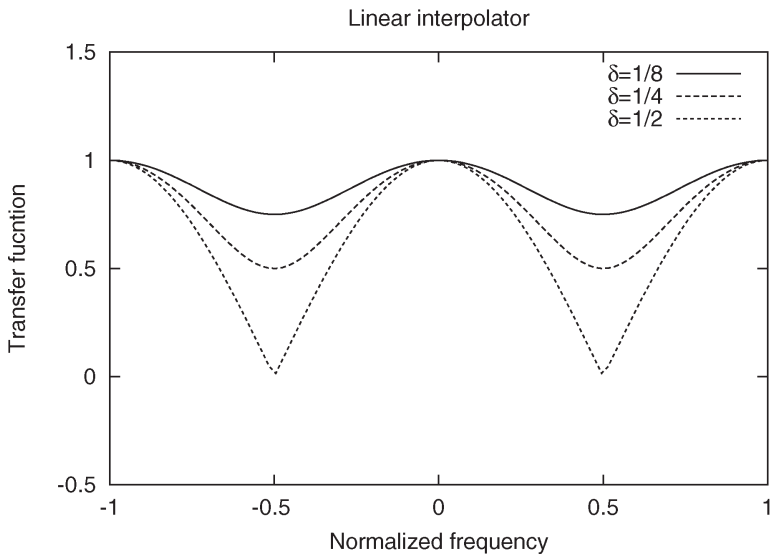

(a)

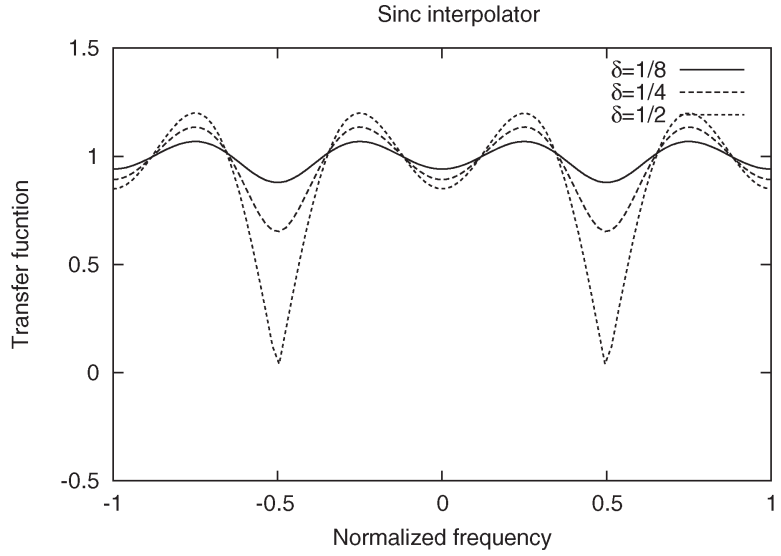

(b)

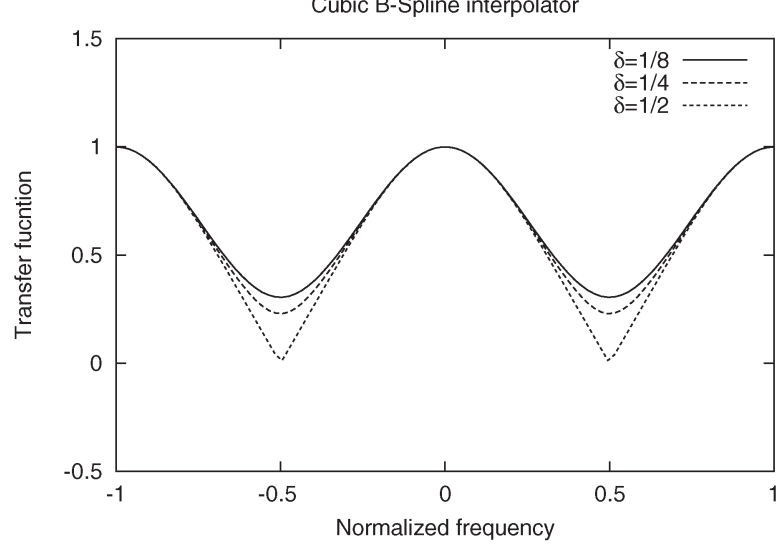

(c)

Fig. 6. Evolution of the blurring effect of the interpolators as a function of the shift. (a) Linear. (b) Truncated sinc. (c) Cubic B-spline.

entropy-based registration measures, when plotted as a function of the geometric transformation, show sudden changes. They show that this registration function depends on the interpolation method in the cases of similar sampling step for the two images to be registered and propose to resample one of the images with a slightly different sampling step. This solution can be applied in the case of global image registration where one looks for a parametric transformation (i.e., affine transformation). In this case, the resampling introduces a scaling effect which can be compensated by the geometric transformation. In the case of disparity-map estimation, the sampling step of the images has to be the same, in order to approximate the deformations by local rigid shifts.

Tsao [6] also analyzes the behavior of mutual-information surfaces for four different interpolators (nearest neighbor, linear, cubic, and Hamming-windowed sinc). He shows the influence of the number of bins used for the estimation of the histograms needed for the computation of the mutualinformation measure. He proposes to blur the histograms and to introduce some jitter in the sampling step. The jitter will produce an effect which is analogous to the resampling proposed by Pluim et al. [5]. One has to note that the blurring of the histograms can only be applied to histogram-based similarity measures. Also, the jitter of the sampling step will introduce local errors which cannot be accepted in subpixel registration.

Ji et al. [7] also analyze these effects and propose other strategies for reducing the artifacts: image oversampling and intensity clustering. The image oversampling produces an effect similar to blurring, and the intensity clustering produces smoother histograms. Once again, this second solution can only be applied to histogram-based similarity measures.

Our theoretical model will show that these interpolation artifacts are not specific to the mutual-information measure, and that, therefore, their origin does not reside in the histogram estimation.

\section{A. Theoretical Model}

In this section, we show that the origin of the observed artifacts is the interpolation procedure used for the subpixel registration. In this procedure, we resample the local image patches in order to measure the similarities for different shifted positions. The resampling is performed by image interpolation. Fig. 5 illustrates the procedure. In order to obtain a shift of $\delta<1$ pixels, we have to estimate the image gray levels at positions which lay between the samples of the image. The image to be resampled $x[n]$ is considered to be the sampled version of an ideal continuous image $x(t)$

$$
x[n]=x(n T)
$$

where $T$ is the sampling step. The shifted image $y[n]$ will be obtained by sampling the same original image $x(t)$ with a shifted sampling grid. Assuming that $x(t)$ was correctly sampled (with respect to the Shannon criterion), we can retrieve 


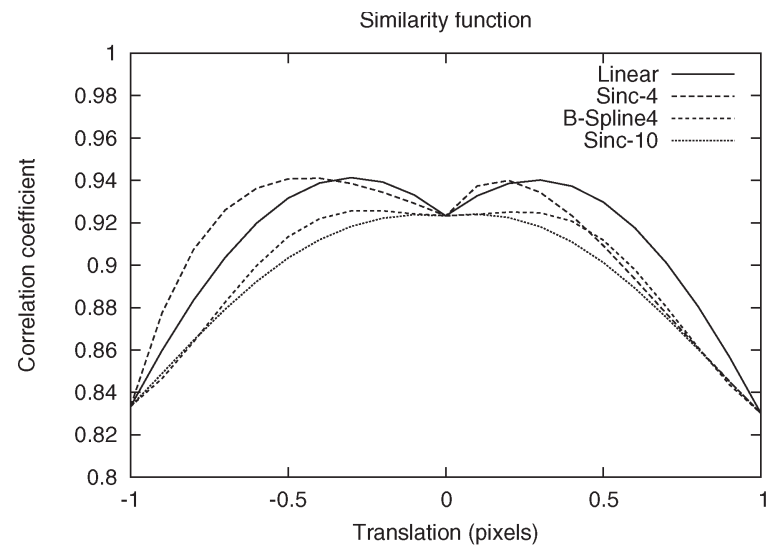

(a)

Fig. 7. Comparison of interpolators.

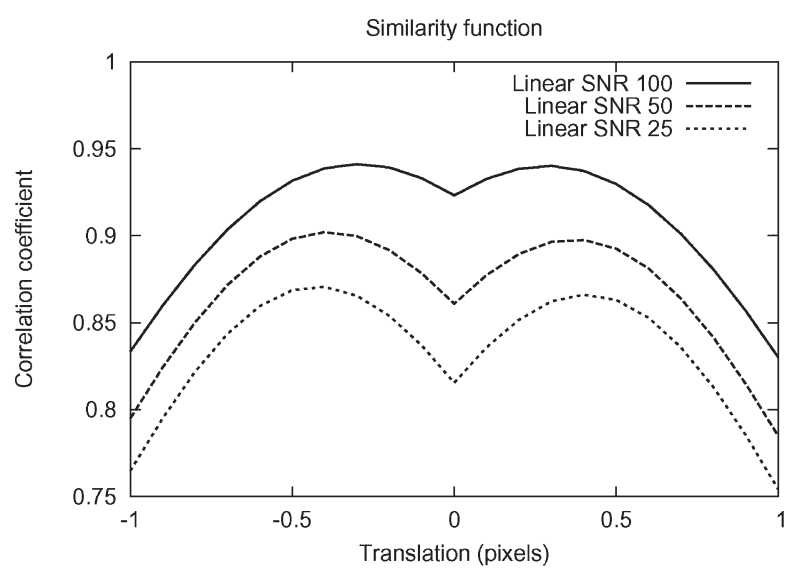

(a)

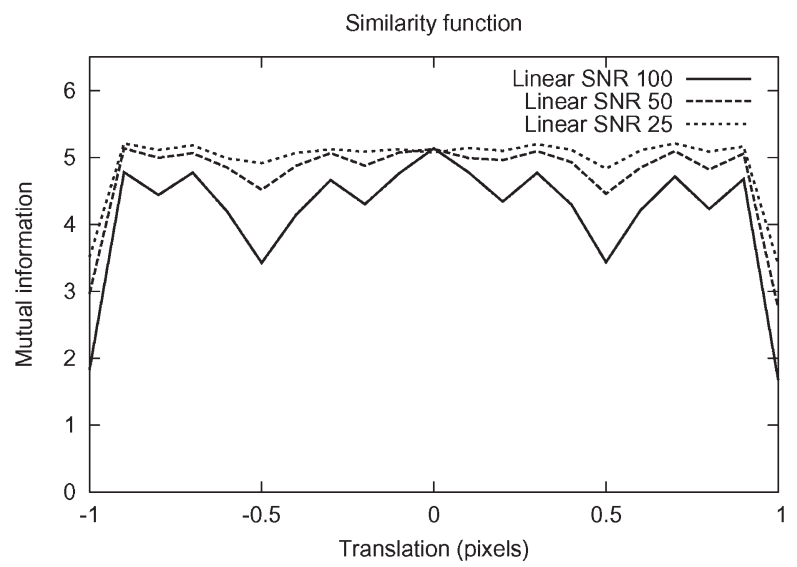

(c)

Fig. 8. Influence of noise level on the registration functions.

$x(t)$ from $x[n]$ by ideal interpolation, i.e., by using a sinc interpolator.

The sinc interpolator has an infinite impulse response. Therefore, approximate interpolators will be used. In order to increase the computation speed, we want to use interpolation filters with a low number of samples.

For a linear interpolator, the interpolated image $y(t)$ for a shift $\delta$ will take the following expression:

$$
y(t)=(1-\delta) x(t-\delta)+\delta x(t+1-\delta)
$$

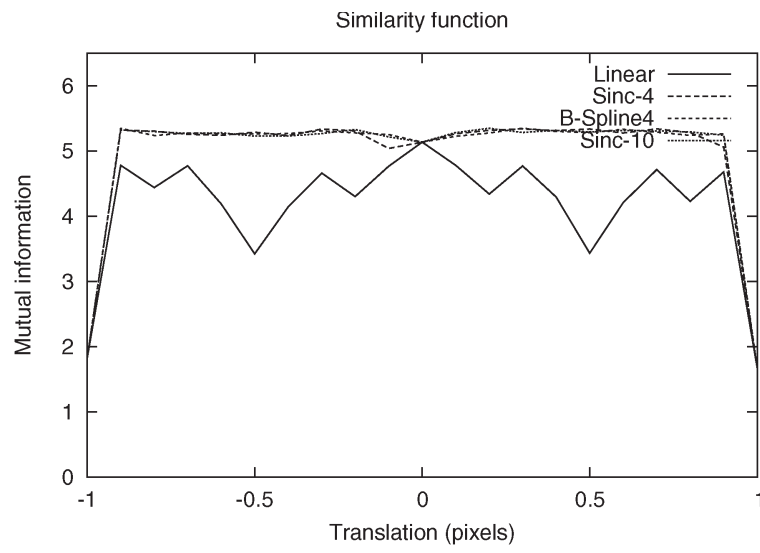

(b)

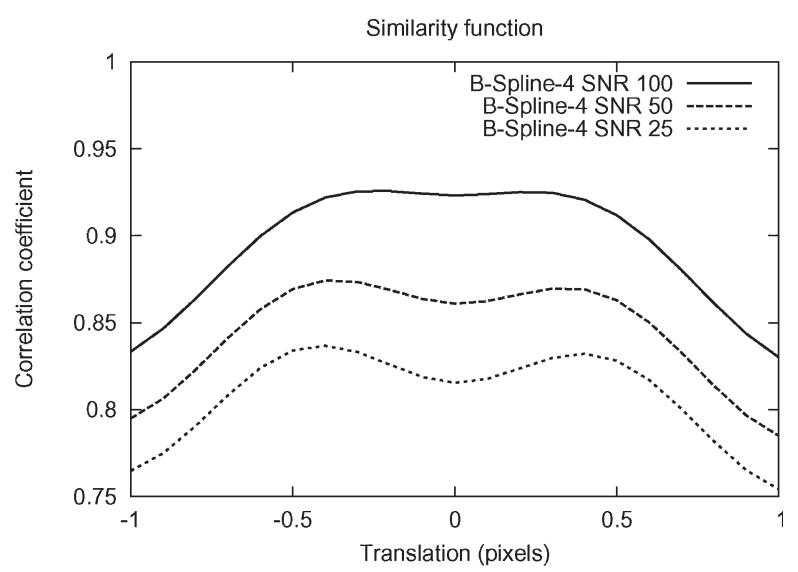

(b)

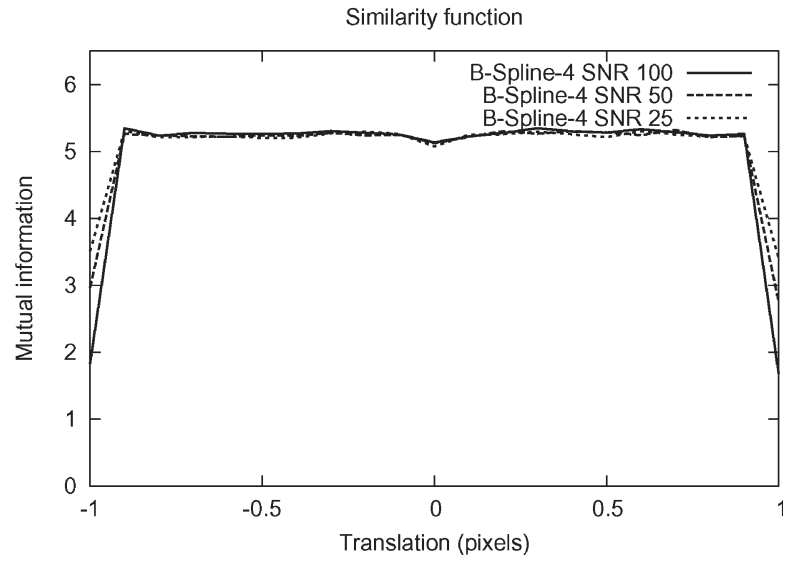

(d)

and its Fourier transform is

$$
Y(f)=X(f)\left[(1-\delta) e^{-j 2 \pi \delta f}+\delta e^{-j 2 \pi(\delta-1) f}\right]
$$

We see that the interpolated signal $y(t)$ is not exactly equal to the original signal $x(t)$ due to the fact that we are not using an ideal interpolator. Instead, we obtain a low-pass filtered version of the original signal. It is interesting to note that the blurring of 


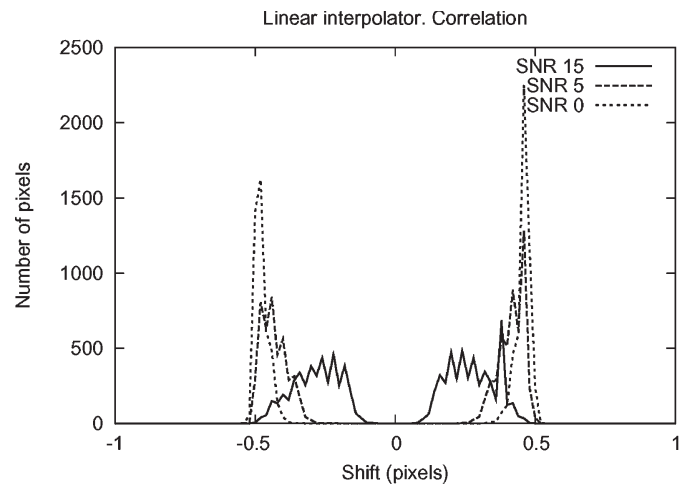

(a)

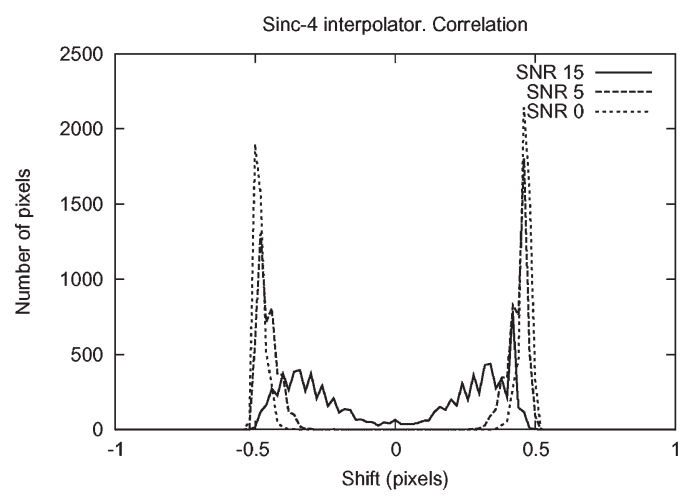

(c)

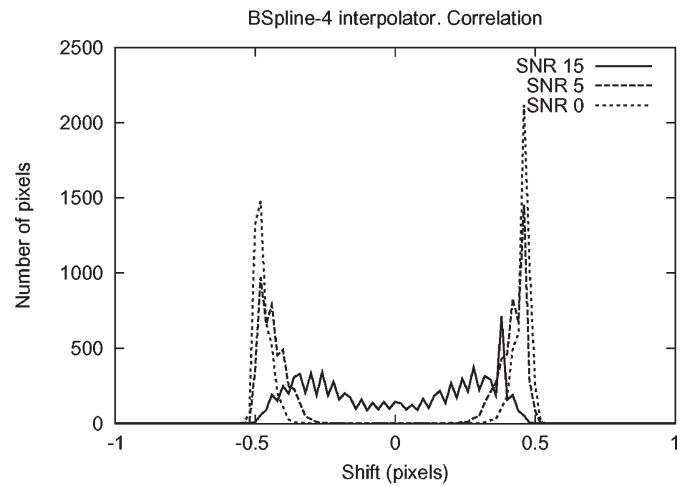

(e)

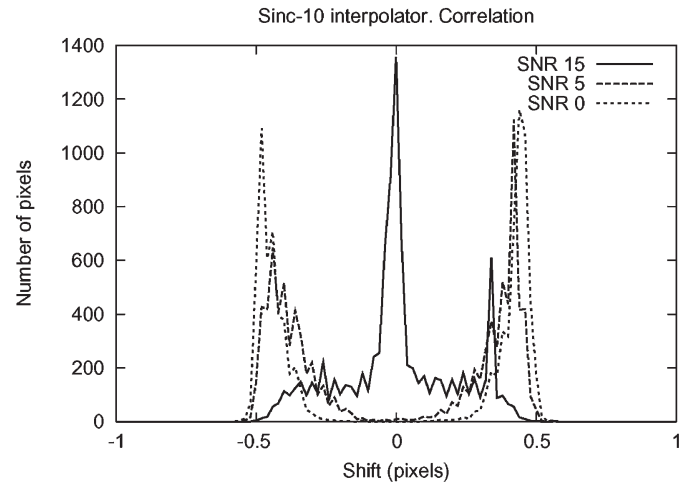

(g)

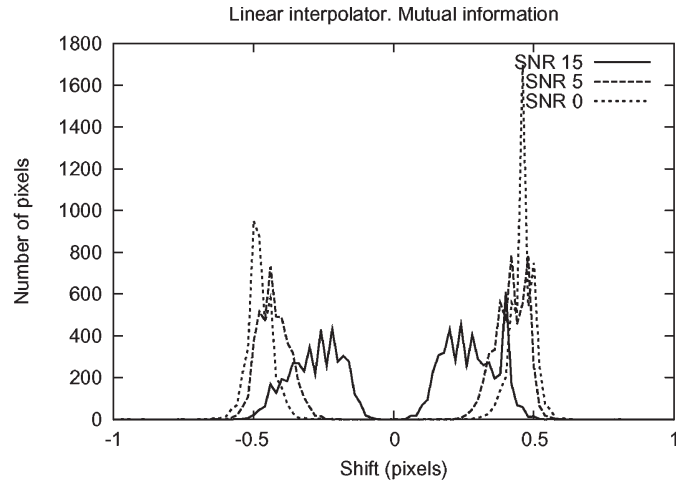

(b)

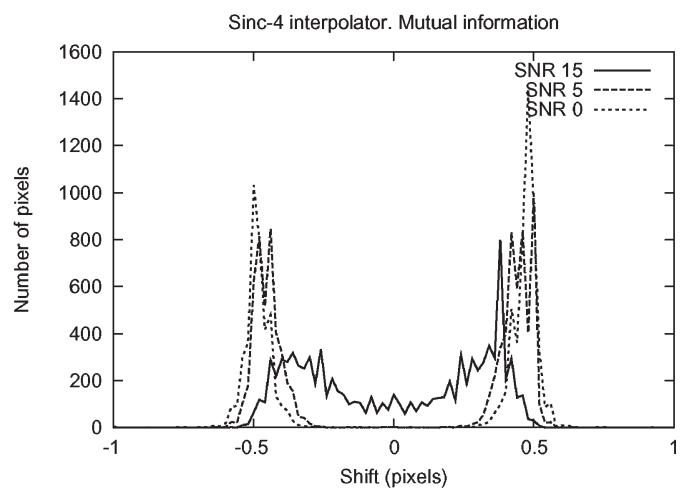

(d)

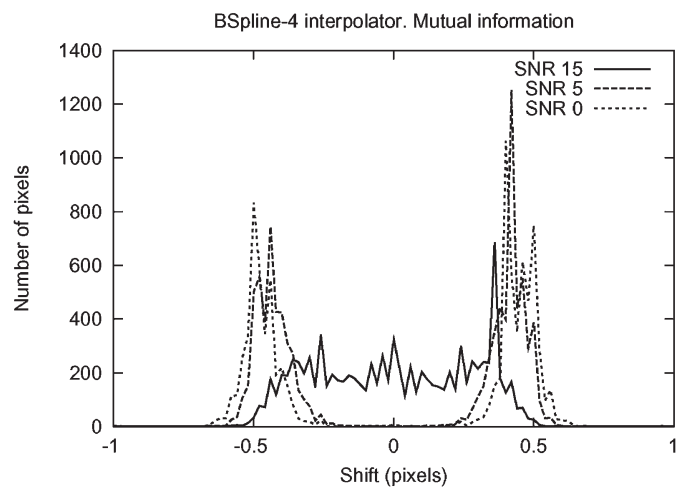

(f)

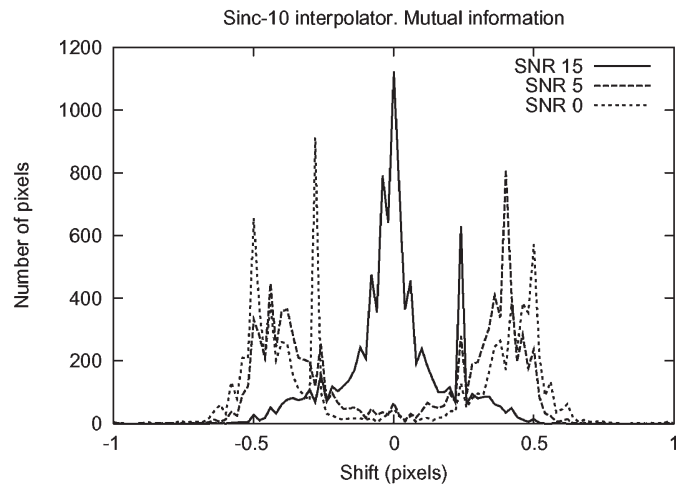

(h)

Fig. 9. Influence of noise level on the estimated shifts.

the image introduced by the interpolation depends on the shift. Fig. 6(a) shows that the blurring effect increases when the shift comes close to half a pixel $(\delta=1 / 2)$.
This means that, in the case of noisy images, the interpolation has a denoising effect, and therefore, it increases the quality of the similarity estimation. Since this blurring is not the same for 


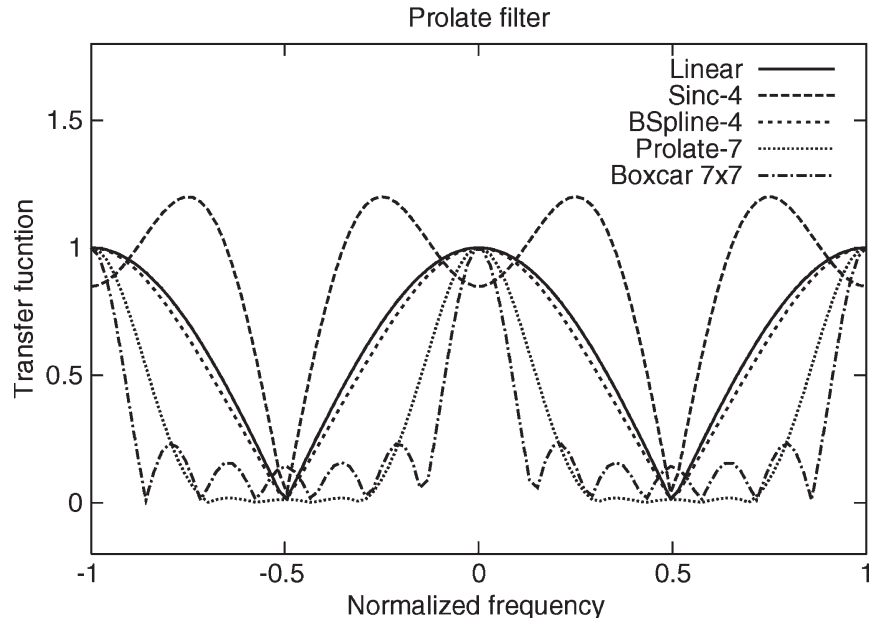

Fig. 10. Frequency response of the smoothing prolate filter compared to different interpolators for $\delta=1 / 2$. The $7 \times 7$ boxcar filter is also shown.

every shift, the similarity surface may show low values for a null shift (no blurring) and higher values for shifts close to half a pixel (strong blurring). It is important to note that the artifacts do not come from the blurring effect itself, but rather from the difference of blurring effect for different shift values.

Its is now interesting to analyze this effect for other interpolators. For the case of an interpolator $c(t)$ truncated to four samples, the Fourier transform of the interpolated signal takes the following expression:

$$
\begin{aligned}
Y(f)=X(f)\left[c(\delta+1) e^{-j 2 \pi(\delta+1) f}+c(\delta) e^{-j 2 \pi \delta f}\right. \\
\left.\quad+c(1-\delta) e^{-j 2 \pi(1-\delta) f}+c(2-\delta) e^{-j 2 \pi(2-\delta) f}\right]
\end{aligned}
$$

For the case of a sinc interpolator, $c(t)=\sin (\pi t) / \pi t$. The frequency response of the interpolator as a function of the shift $\delta$ is shown in Fig. 6(b). We see that the blurring effect is still dependent on the shift, but also that, for such a short filter, the continuous frequency is also filtered. This can produce effects which are worse than the linear interpolator. We will see this in the following sections.

Finally, we analyze the case of a cubic B-spline interpolator [10]. In this case, the filter coefficients take the following expression:

$$
c(t)= \begin{cases}\frac{2}{3}-\frac{1}{2}|x|^{2}(2-|x|), & 0 \leq|x|<1 \\ \frac{1}{6}(2-|x|)^{3}, & 1 \leq|x|<2 \\ 0, & |x|>2\end{cases}
$$

Fig. 6(c) shows the frequency response of the four-sample cubic B-spline interpolator. We can see that the blurring effect remains nearly the same for all shifts.

\section{B. Sensitivity Analysis}

We analyze here the behavior of the different interpolators and their effects on the similarity functions. The similarity function is defined as the value of the similarity measure as a function of the shift. Without loss of generality, we will apply the shifts in only one direction. In this case, the similarity function can be plotted as a one-dimensional (1-D) function.
This analysis will be done for the two similarity measures, the correlation coefficient and the mutual information.

The data used for these experiments are SPOT 4 images which are compared to a noisy version of itself. This allows us to ensure that the images are perfectly coregistered. Additive white Gaussian noise has been added to the secondary image with an SNR of $100 \mathrm{~dB}$.

Fig. 7 shows the similarity functions for four different interpolators, the three studied in Section IV-A, plus a sinus cardinal of length equal to ten samples, which is a better approximation of the ideal one. Even if a similarity function shows only the behavior for a particular pixel, the examples presented here can be considered as examples of what occurs for every pixel in the image. For the case of the correlation coefficient [Fig. 7(a)], we obtain a behavior which could be predicted from the theory presented above. The linear and the sinc- 4 interpolators have strong maxima close to the half-pixel shifts. We can observe that these effects are much weaker for the B-spline interpolator and that they are nearly inexistent for the sinc-10 one.

It is worth to notice that the erroneous maxima are not exactly located on the half-pixel shifts and that they are not symmetrical with respect to the null translation. This is caused by the fact that we are measuring the similarity between an image and its noisy shifted blurred version with a degree of blurring which depends on the shift. The blurring is useful for denoising and thus for increasing the similarity. On the other hand, the shift decreases the similarity because the homologous pixels are further away. Therefore, the combination of these two effects may produce a similarity maximum whose location depends on the local content of the image.

This is the case for the mutual-information plots shown on Fig. 7(b). As discussed in [8], mutual-information peaks have a higher slope than the correlation coefficient ones. That means that the effect of erroneous peaks will only appear for interpolators whose behavior is very sensitive to the shifts. Also, one could expect that the erroneous maxima will appear near the null shift. This is what can be observed in the plots. For the linear interpolator, the peaks appear for about one third of a pixel. We can also observe that, since mutual information is able to measure the dependence in the presence of noise [11], the global maximum is located at zero, even if its value is not much higher that the secondary maxima. For the case of interpolators with a more stable smoothing, one can see that there is no clear peak, meaning that the smoothing effect produces a high value of mutual information even for shifts larger than half a pixel. Also, the flatness of the measure makes it unusable in an optimization problem. Of course, the mutual-information value is low for integer pixel shifts, since no interpolation is applied in this case.

We can also analyze the influence of the noise level on the similarity functions. Fig. 8 shows the behavior of the linear and the B-spline interpolators for the correlation coefficient and the mutual information for different SNR of the secondary image.

In the case of the correlation coefficient, we observe that the relative value of the erroneous peaks with respect to the value at zero is higher for a lower SNR. Of course, the absolute value of the peaks is lower. We also see that the B-spline interpolator is more robust to high noise levels. In the case of mutual information, we observe that the similarity function becomes 


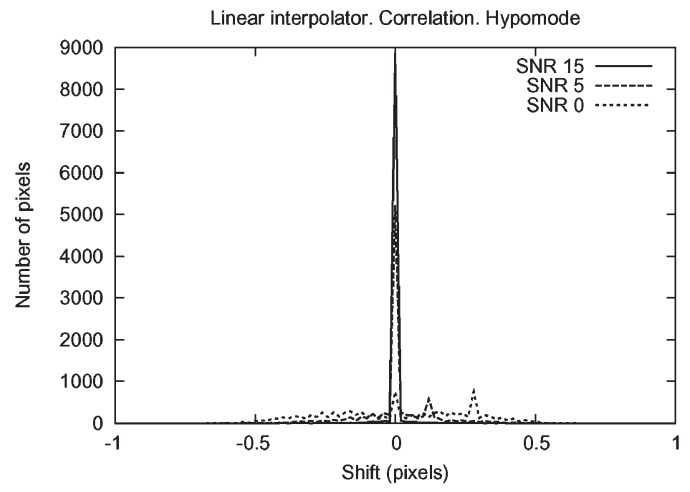

(a)

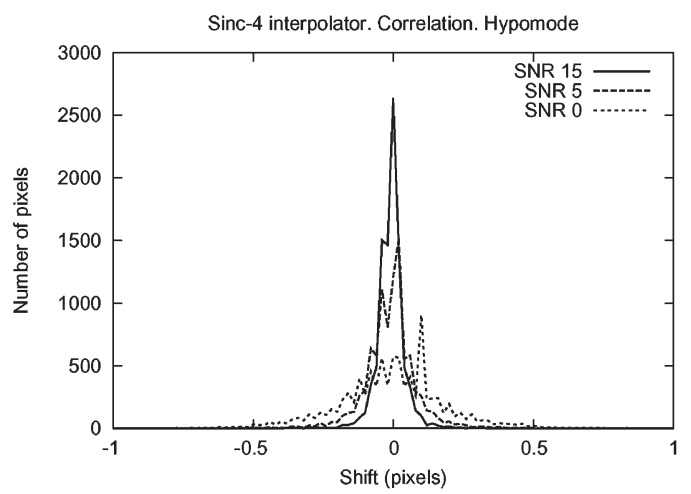

(c)

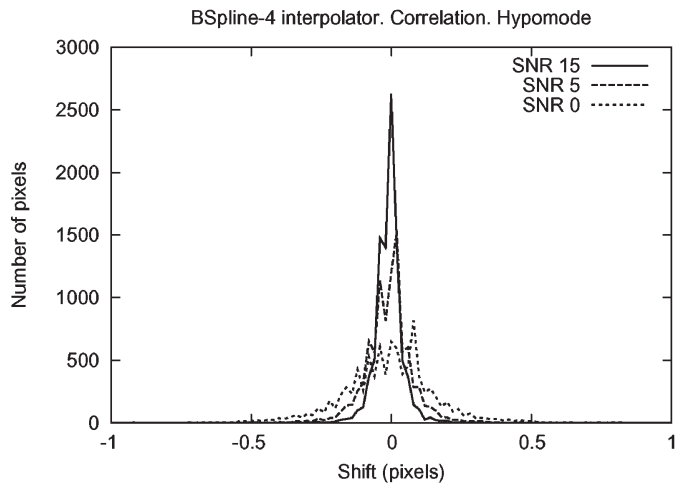

(e)

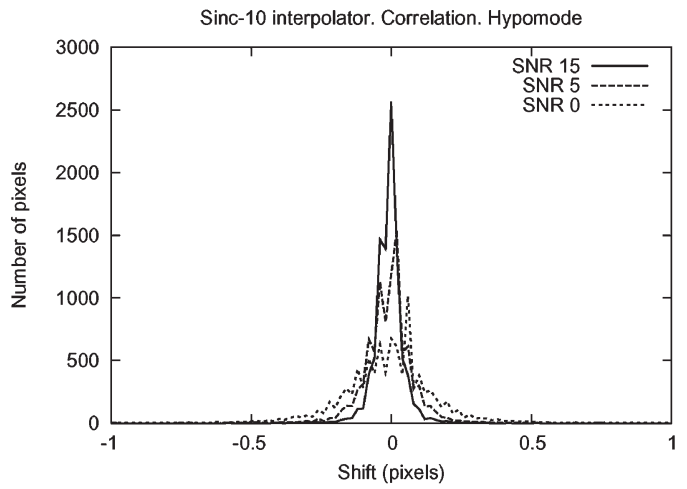

(g)

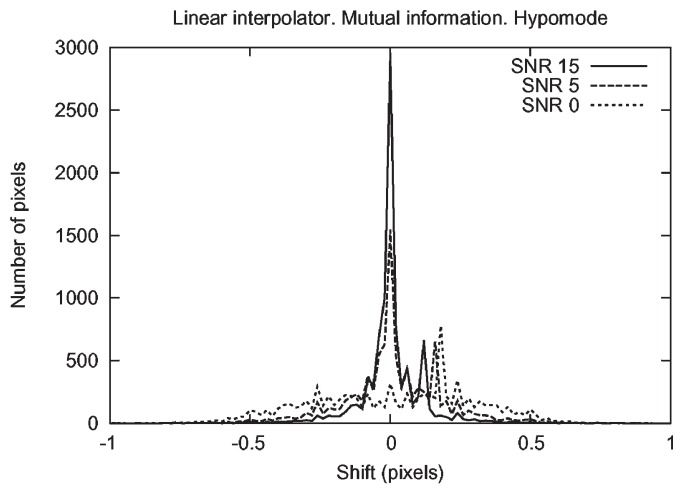

(b)

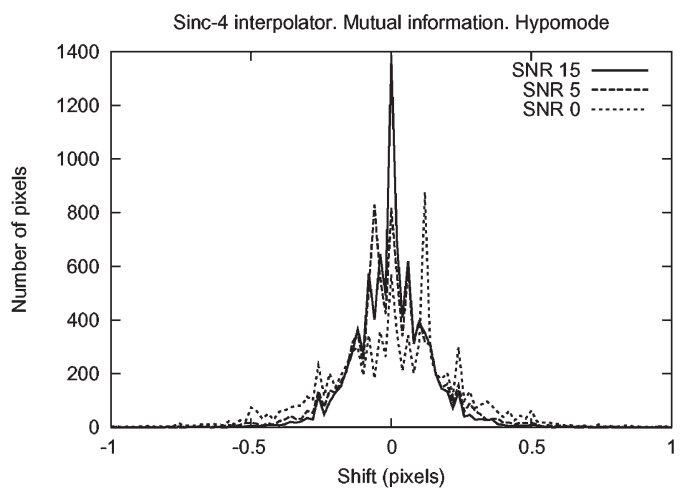

(d)

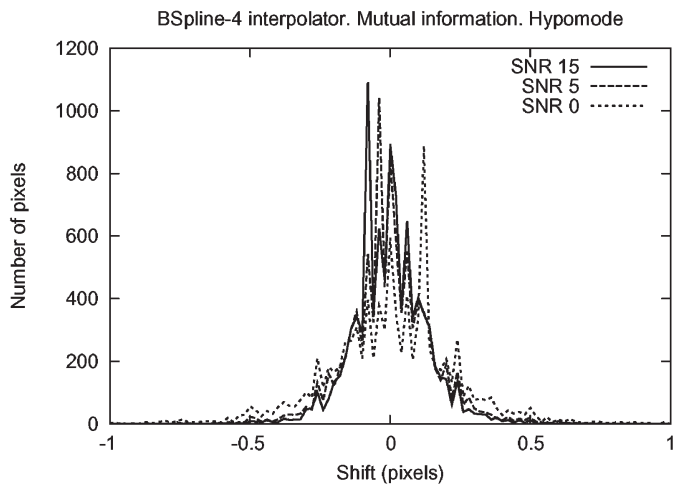

(f)

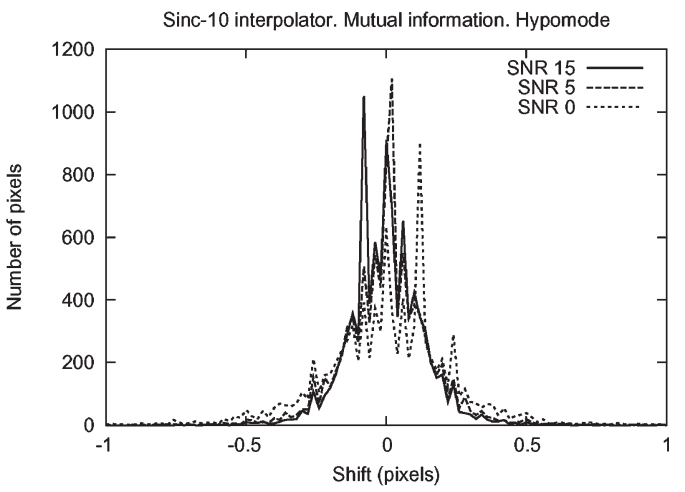

(h)

Fig. 11. Influence of low-pass filtering on the estimated pixel shifts for different noise levels.

flat when the noise increases [Fig. 8(c)]. This is coherent with what we saw in Fig. 7(b) for the most stable interpolators and is also coherent with Fig. 8(d).
Since the registration functions of Figs. 7 and 8 show only the behavior of a selected pixel of the image, it is difficult to infer the global quality of the registration from them. In order 
to study the global quality, we will analyze the histograms of the estimated shifts. We will study the different combinations of interpolators (linear, sinc-4, cubic B-spline, and sinc-10), similarity measures (correlation coefficient and mutual information), and noise level. The results are shown in Fig. 9.

As for the previous simulations, 1-D shifts have been applied. In terms of noise influence, one observes that, the higher the SNR, the lower the number of shifts at a multiple of 0.5 pixels. We also observe that, when the SNR increases, the peaks move close to the null shift.

If we compare the interpolators for a given SNR, say $15 \mathrm{~dB}$, we see that the better the interpolator (linear is worst, then sinc-4, B-spline, and sinc-10 is the best), the higher the number of pixels for which the estimated shift is close to zero, which is the expected value. This is true for both similarity measures.

\section{Attenuation of the Artifacts}

As it has been stated above, we are interested in using short interpolating filters, since the interpolation is performed a high number of times during the similarity optimization procedure. As we have shown above, the interpolation artifacts are produced by the blurring effects of the interpolators. More precisely, the origin of the artifacts is not the blurring effect itself but rather the difference of blurring intensity as a function of the applied shift. We have shown, for instance, that, even if the B-spline interpolator has a stronger blurring effect than the linear interpolator, since its blurring remains nearly constant for all shifts, it has better performances for the disparity-map estimation.

The strategy proposed here for reducing the interpolation artifacts is a very simple one. Since the interpolator is going to introduce a blurring effect, we can smooth the secondary image with a filter whose transfer function is identical (in modulus) to the maximum blurring effect of the interpolator. This can be done in a preprocessing step.

However, when observing Fig. 6, we see that the evolution of the blurring effect may not be only related to the highest frequencies of the signal, and therefore, selecting the transfer function of the preprocessing filter could be tricky.

For instance, choosing a simple boxcar filter for preprocessing can produce artifacts introduced by the secondary lobes of the filter. These lobes come from the windowing used for the truncation of the filter's impulse response.

In order to study the improvement of the subpixel shift estimation for the different interpolators, we choose to use the same smoothing filter for all of them. In order to reduce the secondary lobes of the smoothing filter and to assure a short impulse response, we propose to use a prolate function [12], [13]. The prolate filter is one class of the nonrecursive finite impulse response filters. It is superior to other filters in this class in that it has a maximum energy concentration in the frequency passband and minimum ringing in the time domain.

A prolate filter with seven samples is shown in Fig. 10 and is compared to the maximum smoothing for several interpolators. The frequency response of the $7 \times 7$ boxcar filter, with its secondary lobes, is also shown.

Fig. 11 shows the same kind of analysis as Fig. 9, but with the use of the prolate filter as a preprocessing step for the secondary image. The first remark we can make is that the peaks at multiples of 0.5 pixels have vanished for both similarity measures and for all interpolators. The behavior of the correlation is always better than that of the mutual information.

We also see that, for the high SNR values, the best results are obtained for the linear interpolator. However, for low SNR values, the better the interpolator, the better are the estimated shifts. We can also see that, for the mutual-information case, good results are only obtained with the linear and the sinc-4 interpolators, and for the highest SNR value.

\section{CONCLUSION}

This paper has presented the problem of interpolationinduced artifacts in the procedure of disparity-map estimation used for subpixel image registration. The problem has been introduced with a real case, where the presence of wrongly estimated shifts when a radar image is interpolated have been shown.

A theoretical explanation of the origin of the artifacts has been given, and it demonstrated that the blurring effect of the interpolator, which is dependent on the applied shift, is responsible for the errors observed in the registration functions.

Several interpolators have been compared under different SNR conditions. Finally, it has been shown that a preprocessing step which smoothes the secondary interpolated image can solve the problem. However, attention has to be paid to the choice of the smoothing filter. Indeed, simple filters, as the boxcar one, have to be avoided since they present secondary lobes for the frequencies where the interpolation artifacts occur.

Even if the solution presented here allows for an improvement of the estimation of subpixel-accuracy disparity maps, more work has to be done in order to:

1) analyze the effects of the interpolation for other image modalities, as infrared data, for example;

2) study the influence of image resolution and type of landscape in the quality of the estimated shifts;

3) propose edge-preserving smoothing filters which, combined with different interpolators, could help to attenuate the interpolation artifacts at the same time that they preserve high-frequency content which can be useful for a precise disparity estimation.

\section{REFERENCES}

[1] J. Townshend, C. Justice, C. Gurney, and J. McManus, "The impact of misregistration on change detection," IEEE Trans. Geosci. Remote Sens., vol. 30, no. 5, pp. 1054-1060, Sep. 1992.

[2] G. Cliche, F. Bonn, and P. Teillet, "Integration of the SPOT Pan channel into its spectral mode for image sharpness enhancement," Photogramm. Eng. Remote Sens., vol. 51, pp. 311-316, Mar. 1985.

[3] P. Chavez, S. Sides, and J. Anderson, "Comparison of three different methods to merge multiresolution and multispectral data: TM \& SPOT pan," Photogramm. Eng. Remote Sens., vol. 57, no. 3, pp. 295-303, 1991.

[4] T. Toutin, "Multi-source data fusion with an integrated and unified geometric modeling," EARSeL Adv. Remote Sens., vol. 4, no. 2, pp. 118-129, 1995.

[5] J. P. W. Pluim, J. B. A. Maintz, and M. A. Viergever, "Interpolation artefacts in mutual information-based image registration," Comput. Vis. Image Underst., vol. 77, no. 2, pp. 211-232, Feb. 2000.

[6] J. Tsao, "Interpolation artifacts in multimodality image registration based on maximization of mutual information," IEEE Trans. Med. Imag., vol. 22, no. 7, pp. 854-864, Jul. 2003.

[7] J. X. Ji, H. Pan, and Z.-P. Lang, "Further analysis of interpolation effects in mutual information-based image registration," IEEE Trans. Med. Imag., vol. 22 , no. 9, pp. 1119-1131, Sep. 2003. 
[8] J. Inglada and A. Giros, "On the possibility of automatic multisensor image registration," IEEE Trans. Geosci. Remote Sens., vol. 42, no. 10, pp. 2104-2120, Oct. 2004.

[9] I. Csiszar, "Information type measures of difference of probability distributions and indirect observations," Studia Sci. Math. Hungar., no. 2, pp. 299-318, 1967.

[10] M. Unser, A. Aldroubi, and M. Eden, "B-spline signal processing: Part I-Theory," IEEE Trans. Signal Process., vol. 41, no. 2, pp. 821832, Feb. 1993.

[11] J. Inglada, "Similarity measures for multisensor remote sensing images," in Proc. IEEE Geosci. Remote Sens. Symp., Toronto, ON, Canada, Jun. 24-28, 2002, vol. 1, pp. 104-106.

[12] D. Slepian, "Prolate spheroidal wave functions, Fourier analysis, and uncertainty-V: The discrete case," Bell Syst. Tech. J., vol. 57, no. 5, pp. 1371-1429, May 1978.

[13] J. D. Mathews, J. K. Breakall, and G. K. Karawas, "The discrete prolate spheroidal filter as a digital signal processing tool," IEEE Trans. Acoust., Speech Signal Process., vol. ASSP-33, no. 6, pp. 1471-1478, Dec. 1985.

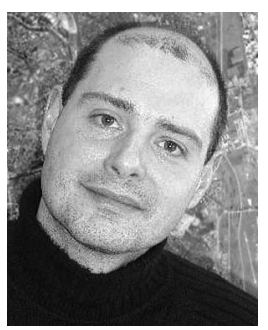

Jordi Inglada received the Telecommunications Engineer degree from both the Universitat Politècnica de Catalunya, Barcelona, Spain, and the École Nationale Supérieure des Télécommunications de Bretagne, Brest, France, in 1997 and the Ph.D. degree in signal processing and telecommunications from the Université de Rennes 1, Rennes, France, in 2000 .

$\mathrm{He}$ has been working at the Centre National d'Etudes Spatiales, Toulouse, France, in the field of remote sensing image processing. He is in charge for the development of image processing algorithms for the operational exploitation of Earth observation images, mainly in the fields of image registration, change detection, and object recognition. He hopes that one day society will be able to fully benefit from the amazing amount of information contained in remote sensing images.

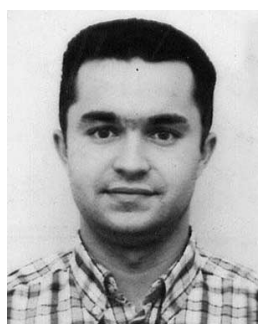

Vincent Muron received the D.E.S.S. degree in remote sensing and digital image processing from Université Paul Sabatier, Toulouse, France, after a degree in physics, in 1998 and the M.Sc. degree in signal and image processing from the École Nationale Supérieure d'Electrotechnique, d'Electronique, d'Informatique d'Hydraulique et des Télécommunications, Toulouse, in 2000.

Since 2000 , he has been working with the Image Processing Office, Communication et Systèmes, Toulouse. His main interests include image registration and image segmentation and classification.

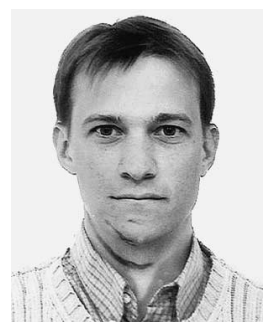

Damien Pichard received the Engineer degree from the École Spéciale de Mécanique et d'Electricité, Paris, France, in 1997 and the Master degree in image and signal processing from the École Nationale Supérieure d'Electrotechnique, d'Electronique, d'Informatique d'Hydraulique et des Télécommunications, Toulouse, France, in 1998.

Since 2000, he has been working with Communication et Systèmes, Toulouse, as a member of the Image Processing Office in the Space Division. He has worked on image compression, image segmentation and classification, and image registration and interpolation. He is currently Responsible of the MEDICIS software development and distribution. This software computes the disparity between pairs of images. This tool is specified by the Centre National d'Etudes Spatiales (CNES), and it is used in the SPOT image quality processing chain by the CNES.

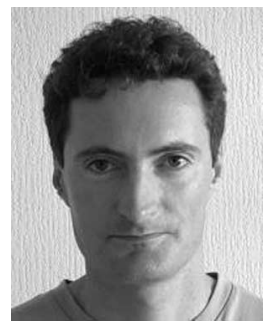

Thomas Feuvrier received the D.E.S.S. degree in remote sensing and digital image processing from Université Paul Sabatier, Toulouse, France, in 1998.

Since 2000, he has been working with Communication et Systèmes, Toulouse, in the field of image processing for space, industrial, and defense applications (image quality assessment, similarity measures, and image registration). At present, he takes an important role in the development of the ORFEO Toolbox, the CNES open source software library for high-resolution remote sensing image processing. 